\title{
Discriminating three motor imagery states of the same joint for brain-computer interface
}

\author{
Shan Guan ${ }^{1}$, Jixian Li ${ }^{\text {Corresp., } 1}$, Fuwang Wang ${ }^{1}$, Zhen Yuan ${ }^{1}$, Xiaogang Kang ${ }^{1}$, Bin Lu ${ }^{1}$ \\ ${ }^{1}$ School of Mechanical Engineering, Northeast Electric Power University, Jilin, China \\ Corresponding Author: Jixian Li \\ Email address: 2201900511@neepu.edu.cn
}

The classification of electroencephalography (EEG) induced by the same joint is one of the major challenges for brain-computer interface $(\mathrm{BCl})$ systems. In this paper, we propose a new framework, which includes two parts, feature extraction and classification. Based on local mean decomposition (LMD), cloud model, and common spatial pattern (CSP), a feature extraction method called LMD-CSP is proposed to extract distinguishable features. In order to improve the classification results, the multi-objective grey wolf optimization twin support vector machine (MOGWO-TWSVM) is applied to discriminate the extracted features. We evaluated the performance of the proposed framework on our laboratory data sets with three motor imagery (MI) tasks of the same joint (shoulder abduction, extension, and flexion), and the average classification accuracy was $91.27 \%$. Further comparison with several widely used methods showed that the proposed method had better performance in feature extraction and pattern classification. Overall, this study can be used for developing high-performance $\mathrm{BCl}$ systems, enabling individuals to control external devices intuitively and naturally. 
1 Discriminating three imagery states of the same joint for brain-computer

2 interface

3

4 Shan Guan, Jixian Li, Fuwang Wang, Zhen Yuan, Xiaogang Kang, Bin Lu

School of Mechanical Engineering, Northeast Electric Power University, Jilin City, Jilin Province, 7 China

9 Corresponding Author:

10 Jixian $\mathrm{Li}$

11 School of Mechanical Engineering, Northeast Electric Power University, Jilin City, Jilin Province, 12 132012, China

13 Email address: 2201900511@ neepu.edu.cn

\section{Abstract}

The classification of electroencephalography (EEG) induced by the same joint is one of the major challenges for brain-computer interface (BCI) systems. In this paper, we propose a new framework, which includes two parts, feature extraction and classification. Based on local mean decomposition (LMD), cloud model, and common spatial pattern (CSP), a feature extraction method called LMD-CSP is proposed to extract distinguishable features. In order to improve the classification results, the multiobjective grey wolf optimization twin support vector machine (MOGWO-TWSVM) is applied to discriminate the extracted features. We evaluated the performance of the proposed framework on our laboratory data sets with three motor imagery (MI) tasks of the same joint (shoulder abduction, extension, and flexion), and the average classification accuracy was $91.27 \%$. Further comparison with several widely used methods showed that the proposed method had better performance in feature extraction and pattern classification. Overall, this study can be used for developing high-performance BCI systems, enabling individuals to control external devices intuitively and naturally.

\section{Introduction}

$\mathrm{BCI}$ is a technology that can directly establish communication and control between human brain and computer or other electronic equipment (Li et al., 2020; Park \& Chung, 2020). BCI technology is widely applied in medical rehabilitation, smart home, entertainment, military, and other fields. At present, the EEG traces include sensorimotor rhythms (SMR) (Blankertz et al., 2010), slow cortical potential (SCP) (Hinterberger et al., 2004), event-related potential (ERP) (Delgado et al., 2020), and visual-evoked potential (VEP) (Zhou et al., 2020). SMR is induced by motor imagery without external stimulation, so it is widely used in BCI systems. However, the EEG is a non-stationary, non-linear, and noisy signal. And, it is easily interfered by the environment when it is recorded, the signal-to-noise ratio is low, which makes it more challenging to identify the EEG of MI tasks.

In the past few decades, researchers have proposed various feature extraction methods and classification algorithms to classify MI tasks efficiently. The most classical feature extraction methods include wavelet transform (WT) (You, Chen \& Zhang, 2020), empirical mode 
42 decomposition (EMD) (Taran et al., 2018), common spatial pattern (CSP) (Yang et al., 2016;

43 Selim et al., 2018), and filter-bank CSP (FBCSP) (Ang et al., 2008; Wang et al., 2020). The

44 widely used classification algorithms include linear discriminant analysis (LDA) (Aljalal, Djemal

45 \& Ibrahim, 2019), extreme learning machine (ELM) (Rodrguez-Bermudez, Bueno-Crespo \&

46 Martinez-Albaladejo, 2017), k-nearest neighbors (KNN) (Bashar, Hassan \& Bhuiyan, 2015), support vector machine (SVM) (Selim et al., 2018;) and least squares support vector machine (LS-SVM) (Taran et al., 2018; Taran \& Bajaj, 2019). Malan and Sharma applied dual-tree complex wavelet transform (DTCWT) to extract time, frequency, and phase features of left and right hand MI EEG signals, and classified them using SVM with an average accuracy of $80.7 \%$ (Malan \&Sharma, 2019). However, it's usually hard to select an appropriate wavelet basis function. Taran et al. employed EMD to extract MI features of left and right hands, and classified them by using LS-SVM with an average accuracy of $97.56 \%$ (Taran et al., 2018). The weakness of that study is that it ignored the endpoint effect and mode mixing phenomenon in the EMD process. Miao et al. classified left and right hand movements with an average accuracy of $86.41 \%$, by using sparse representation of CSP features (Miao, Wang \& Liu, 2017). Kumar et al. proposed a parameter tuning algorithm to improve the performance of CSP by selecting the optimal filter parameters (Kumar \& Sharma, 2018). That study reported an average error recognition rate of $10.19 \%$ on BCI Competition III Dataset IVa (right hand and foot). Recently, Kumar et al. used genetic algorithm (GA) for adaptive filtering, combined CSP and long short-term memory network (LSTM) for feature extraction, and applied SVM for classification (Kumar, Sharma \& Sharma, 2021). It should be noted that these studies focused on binary problems. The aforementioned methods have achieved good recognition results for the classification of the left hand, right hand, foot, and tongue MI tasks. When imagining movements of different limbs, event-related de-synchronization/event-related synchronization (ERD/ERS) will occur in the corresponding areas of the motor cortex. This phenomenon can be recorded by the different electrodes overlying the motor cortex, so BCI systems can efficiently identify MI tasks within different limbs.

However, ERD/ERS induced by MI tasks of the same limb usually occurs in the adjacent region of the motor cortex, which makes it exceedingly difficult to detect the MI tasks within the same joint. Recently, a small number of researchers have conducted preliminary explorations of the recognition of MI tasks within the same joint. For instance, Vuckovic and Sepulveda employed Gabor coefficients calculated from independent components as features and Elman's neural networks as the classifier, the average recognition accuracy of wrist extension and flexion MI was $67.5 \%$ (Vuckovic \& Sepulveda, 2012). As we all know, that accuracy cannot meet the working requirements of BCI systems. Edelman et al. used EEG source imaging technology to identify 4-class MI tasks (wrist flexion, extension, pronation, and supination) with the recognition accuracy of each class exceeding 79.00\% (Edelman, Baxter \& He, 2016).

From the above analysis, we can see that most existing studies focus on the MI tasks of different limbs, which have achieved good recognition results. However, these methods can be 
83 Therefore, this paper proposes a method to decode the various MI movements of the same joint using LMD-CSP and MOGWO-TWSVM. Firstly, LMD is used to decompose the preprocessed MI EEG into a series of product functions (PFs). According to the entropy (En) and super entropy $(\mathrm{He})$ of the cloud model, the real PF components are selected. Secondly, the selected PFs of each channel are reconstructed into a new signal matrix, then feature vectors are extracted by using the CSP. Finally, TWSVM optimized by the MOGWO algorithm is applied to discriminate the extracted features. The proposed framework was verified using our laboratory data sets, which include shoulder abduction, extension, and flexion MI tasks. There were two reasons why we chose these movements. First, these movements were part of the exercise for stroke patients. Second, these movements could intuitively control the robotic arm. In addition, we compared the LMD-CSP and MOGWO-TWSVM with several widely used feature extraction methods and classifiers, respectively. The conventional feature extraction methods include time domain parameters (TDP) (Tavakolan et al., 2017), CSP (Selim et al., 2018), FBCSP (Ang et al., 2008), and common spatial pattern based on empirical mode decomposition (EMD-CSP) (Wang et al., 2008). The traditional classifiers include LDA, ELM, KNN, SVM, and LS-SVM. Those comparisons could confirm the quality of the proposed method.

\section{Materials \& Methods}

\subsection{EEG signal acquisition}

Emotive Epoc+ was employed to record the EEG signals. It includes 14 effective electrodes (AF3, F7, F3, FC5, T7, P7, O1, O2, P8, T8, FC6, F4, F8, and AF4) and 2 reference electrodes (CMS, $\mathrm{DRL})$. The sampling rate is $128 \mathrm{~Hz}$. The layout of electrodes is according to the standard international 10-20 system. The equipment and electrodes arrangement are shown in Fig. 1.

Seven right-handed subjects (five males and two females aged from 23 to 28), who were in good condition both psychologically and physically, were randomly selected to participate in this experiment. All subjects had given written informed consent before the experiment. The study was approved by the Scientific Research Ethics and Technology Safety committee of Northeast Electric Power University.

In the experiment, the subjects were required to perform 3-class MI tasks (shoulder abduction, extension, and flexion) according to prompts. Figure 2 shows the experimental paradigm for the motor imagery (MI) tasks. At the start of each trial, the subjects naturally placed their hands and kept relaxed. At time point $\mathrm{t}=1 \mathrm{~s}$, the monitor showed a cross ' + ', and presented a short beep tone to raise the subject's attention. At $\mathrm{t}=3 \mathrm{~s}$, an arrow pointed to the left, right, or top at random, was shown to indicate the subjects to imagine the corresponding movement (shoulder abduction, extension, or flexion movement). And the arrow disappeared after 1.25 seconds. At $\mathrm{t}=7 \mathrm{~s}$, the subjects stopped motor imagination. The next trial began after 2 seconds. For training classifiers, 60 trials per subject were collected in total (20 trials per class).

\subsection{EEG preprocessing}

Firstly, the raw EEG data were filtered between $8 \mathrm{~Hz}$ and $30 \mathrm{~Hz}$ by a 5 th order zero-phase Butterworth filter to remove DC drift and high-frequency noise. Secondly, the automatic artifact removal (AAR) toolbox was used to remove electrooculogram (EOG) and electromyogram 
123 (EMG) artifacts. Thirdly, the common average reference (CAR) was used to reduce the

124 background noise. The CAR method is the selected channel minus the average of all electrodes.

125 Finally, the denoised EEG data were processed by mirror extending technology to eliminate the

126 influence of the endpoint effect in the LMD process. The extended sequence is defined as follows:

$$
x_{i}(t)=\left[s_{i}(n), s_{i}(n-1), \cdots, s_{i}(1), s_{i}(1), \cdots, s_{i}(n), s_{i}(n), \cdots, s_{i}(1)\right]^{\mathrm{T}}
$$

where $s_{i}(c)$ represents the denoised EEG signal of the $i$ th electrode.

\subsection{Research on feature extraction method based on LMD-CSP}

130 LMD is a new adaptive time-frequency analytical algorithm first proposed by Smith et al. in 2005 (Smith, 2005). When compared with EMD, LMD can better reduce the mode mixing phenomenon and has lower computational complexity and higher decomposition speed (Zhang \&

PFs and a residual component, and the expression is as follows:

$$
x_{i}(t)=\sum_{j=1}^{k} h_{i j}(t)+u_{i}(t)
$$

135

136

137

138

139

140

141

142

143

where $h_{i j}(t)$ is the $j$ th PF obtained from the $i$ th electrode, and $u_{i}(t)$ represents the residual component.

2.3.2 Selection of Product Functions Based on the Cloud Model

The cloud model is an uncertainty transformation model based on fuzzy set theory and probability theory, which can achieve the transformation between a qualitative concept and its quantitative data. It expresses the qualitative concept through the three digital characteristics $\{\mathrm{Ex}$, En, He\}. Expectation (Ex) is the central value of a concept, entropy (En) represents the randomness of a qualitative concept, and super entropy $(\mathrm{He})$ is the dispersion degree of a concept (Wang et al., 2019).

$$
\begin{gathered}
\mathrm{Ex}=\frac{1}{n} \sum_{i=1}^{n} x_{i} \\
\mathrm{En}=\sqrt{\frac{\pi}{2}} \times \frac{1}{n} \sum_{i=1}^{n}\left|x_{i}-\mathrm{Ex}\right| \\
\mathrm{He}=\sqrt{S-\mathrm{En}^{2}}
\end{gathered}
$$

where $S$ is the second-order central moment of $x_{i}, x_{i}(i=1,2, \cdots, n)$ represents the quantitative value of n cloud-droplets.

En and He can express the degree of complexity. The larger the values of En and He, the more complex the signals, and vice versa. Since the structure of the real EEG components is generally complicated, the En and He of the real PFs are larger than those of false PFs. Therefore, the parameters En and He of the cloud model are used to select the effective PFs. In our study, through analyzing a large amount of experimental data, we selected the PF1 component as the effective component for subsequent processing. The details of the effective PF selection can be found in Supplemental Materials. For the recognition of other data sets, we need to employ the entropy (En) and super entropy (He) of the cloud model to select effective PF components again.

In this research, the experimental data of all channels were decomposed by LMD in turn, 
$156 R^{\mathrm{M} \times \mathrm{N}}$, where $\mathrm{M}$ is the order of the selected PFs, and $\mathrm{N}$ represents the sampling point of PF. In the 157 next step, CSP was used to extract the spatial features of $X$.

158 2.3.3 Common Spatial Pattern

159 The goal of CSP is to design a spatial filter which can maximize the variance of two kinds of 160 motor imagery EEG data. Because CSP is based on the simultaneous diagonalization of a 2-class covariance matrix, it can only be used in binary problems. Therefore, we applied the one-versusone (OVO) scheme to LMD-CSP for the multiclass problem, so that a $k$-class problem was transformed into $k(k-1) / 2$ binary class problems. For the signal matrix $X_{i}$ of the $i$ th experimental data, the covariance matrix is calculated as follows:

$$
R=\frac{X_{i} X_{i}^{\mathrm{T}}}{\operatorname{trace}\left(X_{i} X_{i}^{\mathrm{T}}\right)}
$$

165

where "T" represents the transpose operator, and trace $(\cdot)$ means to find the trace of a matrix.

For the binary class MI tasks (i.e., shoulder abduction and extension), we calculated the covariance matrix over the trails of each class, and averaged them to obtain the mean covariance matrix $R_{\mathrm{A}}$ and $R_{\mathrm{E}}$. Then the $R_{\mathrm{A}}$ and $R_{\mathrm{E}}$ were transformed to obtain the spatial filter $W_{\mathrm{AE}}$ (in this paper, $W_{\mathrm{AE}} \in \mathrm{R}^{8 \times 14}$ ) (Liu et al., 2012). Combined with the OVO scheme, we obtained three spatial filters ( $W_{\mathrm{AE}}, W_{\mathrm{AF}}$ and $W_{\mathrm{EF}}$ ). Then the three spatial filters were spliced vertically to gain a global spatial filter. The global spatial filter is as follows:

$$
W=\left[W_{\mathrm{AE}} ; W_{\mathrm{AF}} ; W_{\mathrm{EF}}\right]
$$

where $W_{\mathrm{AE}}$ represents the spatial filter between abduction and extension, $W_{\mathrm{EF}}$ represents the spatial filter between extension and flexion, and $W_{\mathrm{AF}}$ represents the spatial filter between abduction and flexion.

For a single trial data, we obtained the LMD-CSP projection matrix $Z=W X_{i}$. The $p$ th row of $Z$ is denoted as $Z_{p}$. The required characteristics can be obtained by:

$$
f_{p}=\log \left(\frac{\operatorname{var}\left(Z_{p}\right)}{\sum_{k=1}^{g} \operatorname{var}\left(Z_{k}\right)}\right)
$$

177

where $p=1,2, \cdots, \mathrm{g}$. The features obtained by LMD-CSP are denoted as $F=\left[f_{1}, f_{2}, \cdots, f_{\mathrm{g}}\right]$.

Figure 3 shows the distributions of the most significant two LMD-CSP features from subjects 1 . Obviously, the features of different MI tasks extracted by LMD-CSP are highly distinguishable and these are easy to separate.

\subsection{Research on Classification Method Based on MOGWO-TWSVM}

\subsubsection{Twin Support Vector Machine}

TWSVM, first proposed by Jayadeva et.al, is a new machine learning method based on traditional SVM (Jayadeva, Khemchandani \& Chandra, 2007). TWSVM aims to construct a hyperplane for each class. It requires that each hyperplane is close to the corresponding class samples as possible, and far away from the other class samples as possible. TWSVM has higher training speed and generalization ability than SVM because the former solves two small quadratic programming problems (QPPs) to construct the hyperplanes like SVM, and the constraint condition of a QPP is only related to one class of samples. 
190

191

192

193

194

195

196

Suppose that training samples of class 1 are denoted as $A=\left[x_{1}{ }^{(1)}, x_{2}{ }^{(1)}, \cdots, x_{\mathrm{m} 1}{ }^{(1)}\right]^{\mathrm{T}} \in R^{\mathrm{m} 1 \times \mathrm{g}}$, where $x_{j}{ }^{(i)} \in R^{\mathrm{g}}$ represents the $j$ th sample of class $1, m 1$ represents the number of samples.

Similarly, training samples of class 2 are denoted as $B \in R^{\mathrm{m} 2 \times \mathrm{g}}$. When training the TWSVM classifier between class 1 and class 2, two nonparallel hyperplanes are obtained as follows:

$$
\begin{aligned}
& K\left(x^{T}, C^{T}\right) w_{1}+b_{1}=0 \\
& K\left(x^{T}, C^{T}\right) w_{2}+b_{2}=0
\end{aligned}
$$

where $C^{\mathrm{T}}=\left[A^{\mathrm{T}} B^{\mathrm{T}}\right]^{\mathrm{T}}, w_{1}$ and $w_{2}$ are two normal vectors of hyperplanes, $b_{1}$ and $b_{2}$ are the bias vectors.

TWSVM constructs the two hyperplanes by solving the following optimization problems:

$$
\begin{aligned}
& \min _{w^{(1)}, b^{(1)}, \xi^{(2)}} \frac{1}{2}\left\|K\left(A, C^{T}\right) w^{(1)}+e_{1} b^{(1)}\right\|^{2}+c_{1} e_{2}^{T} \xi^{(2)} \\
& -\left(K\left(B, C^{T}\right) w^{(1)}+e_{2} b^{(1)}\right) \geq e_{2}-\xi^{(2)}, \xi^{(2)} \geq 0 \\
& \min _{w^{(2)}, b^{(2)}, \xi^{(1)}} \frac{1}{2}\left\|K\left(B, C^{T}\right) w^{(2)}+e_{2} b^{(2)}\right\|^{2}+c_{2} e_{1}^{T} \xi^{(1)} \\
& -\left(K\left(A, C^{T}\right) w^{(2)}+e_{1} b^{(2)}\right) \geq e_{1}-\xi^{(1)}, \xi^{(1)} \geq 0
\end{aligned}
$$

197

198

199

200

201

202

203

204

205

206

207

208

209

210

211

212

213

214

where $c_{1}$ and $c_{2}$ are penalty parameters, $e_{1}$ and $e_{2}$ are column vectors of ones.

In this study, we established a classifier for each binary class, so $k(k-1) / 2$ TWSVM classifiers were constructed. In the process of classification, the feature vectors of each MI task were input into the classifiers, and the final result was obtained by voting. The penalty parameters $c_{1}$ and $c_{2}$ of the TWSVM were set by the following MOGWO.

\subsubsection{Multi-objective Grey Wolf Optimizer}

MOGWO, proposed by Mirjalili et al., is a new swarm intelligence optimization algorithm based on the conventional grey wolf optimizer algorithm (Mirjalili et al., 2016). When compared with other multi-objective optimization algorithms, MOGWO has higher convergence and coverage (Dilip et al., 2018). In MOGWO, the location of each gray wolf is denoted as a solution, and the first three best solutions are denoted as the alpha $(\alpha)$ wolves, beta $(\beta)$ wolves, and delta $(\delta)$ wolves, the other candidate solutions are omega $(\omega)$ wolves. In the iterative process, $\omega$ wolves are led by $\alpha$ wolves, $\beta$ wolves and $\delta$ wolves to find global optimal solutions.

MOGWO uses an external archive to store and update the non-dominated Pareto optimal solutions. At the same time, it also employs a leader selection mechanism to search for the least crowded segments from the archive, and three non-dominated solutions of the segments are used as $\alpha, \beta, \delta$ wolves by a roulette-wheel method. The location of each search agent is updated as follows:

$$
\begin{gathered}
\overrightarrow{D_{i}}=\left|\overrightarrow{C_{i}} \cdot \overrightarrow{X_{i}}(t)-\vec{X}(t)\right|, i \in(\alpha, \beta, \delta) \\
\overrightarrow{X_{i}}(t)=\overrightarrow{X_{i}}(\mathrm{t})-\overrightarrow{A_{i}} \cdot \overrightarrow{D_{i}}, i \in(\alpha, \beta, \delta) \\
\vec{X}(t+1)=\frac{\overrightarrow{X_{\alpha}}(t)+\overrightarrow{X_{\beta}}(t)+\overrightarrow{X_{\delta}}(t)}{3}
\end{gathered}
$$

215 where the coefficient vector $A \vec{A}$ and $\vec{C}$ are calculated as follows:

$$
\vec{A}=2 \vec{a} \times \overrightarrow{r_{1}}-\vec{a}
$$




$$
\vec{C}=2 \cdot \overrightarrow{r_{2}}
$$

216 where the parameter $\vec{a}$ is decreased from 2 to 0 in the iterative process, and $\overrightarrow{r_{1}}, \overrightarrow{r_{2}}$ are random

217 vectors in $[0,1]$.

218 2.4.3 Multi-objective Function

219 The average recognition accuracy is usually employed to measure classification performance.

220 However, it only quantifies the overall classification performance and ignores evaluating the

221 classification results of each class. Therefore, we applied the mean recognition accuracy and the

222 recognition of each class as objective functions to evaluate the candidate solutions generated by

223 the MOGWO algorithm. The objective function can be expressed as:

$$
\begin{gathered}
\text { Accuracy }=\frac{\sum_{i=1}^{3} N C_{i}}{\sum_{i=1}^{3} N C_{i}+\sum_{i=1}^{3} N E_{i}} \\
C R_{i}=\frac{N C_{i}}{N C_{i}+N E_{i}}, i=1,2,3
\end{gathered}
$$

224 where Accuracy is the mean recognition accuracy, $C R_{i}$ represents the recognition accuracy of $i$ th

225 class, $N C_{i}$ is the number of $i$ th class correctly distinguished samples, $N E_{i}$ is the number of $i$ th

226 class wrongly distinguished samples.

\section{$227 \quad 2.5$ Statistical Analysis}

228 In this study, the one-way analysis of variance (ANOVA) method was used to detect the

229 significant effect of the different methods on classification accuracy. If this ANOVA is

230 significant, we would further use pair-wise t-tests to identify significant differences in recognition

231 results between the proposed method and the other compared methods. It can be considered that

232 there is a significant difference in the classification effect between the two algorithms when the

233 calculated $p$-value is less than 0.05 .

\section{Results}

\subsection{Experimental Results}

236

237

In this research, we employed the Gaussian kernel function with 5-fold cross-validation to search

238 for optimal parameters and obtain the required experimental results. MOGWO was applied to optimize a Gaussian kernel function parameter $\lambda$ and two penalty parameters $c_{1}$ and $c_{2}$ of the classifier model. And the parameter $\lambda$ was selected from 0.001 to 8 , the range of the parameters $c_{1}$ and $c_{2}$ were $[0.01,8]$. The MOGWO parameters used in this paper are listed in Table 1 , and the details of the parameters are given in document (Mirjalili et al., 2016). The Pareto optimal set obtained by MOGWO and the corresponding parameters of the proposed classifier model from subject 4 are given in Table 2. Through analyzing the Pareto optimal set, we selected the optimal solution to obtain the required final recognition accuracy. The optimal solution of subject 4 is marked in Table 2.

Next, we obtained the Pareto optimal set of the remaining subjects through the above steps and the required recognition accuracy of each class and the required average recognition accuracy we got is shown in Fig. 4. We obtained the highest classification accuracy of $98.33 \%$ from 
249 subject 7, and the average classification accuracy of all subjects is $91.27 \%$. Besides, we

250 calculated precision and recall to quantify the classification results. The obtained results are listed

251 in Table 3. In order to intuitively show the experimental results of 3-class MI tasks, we drew the

252 average confusion matrix of all subjects for the proposed method. As can be seen from Fig. 5,

253 shoulder abduction shows the best classification accuracy (95.71\%). In the meantime, through

254 analyzing the classification accuracy of each class MI task, we find that the recognition accuracy

255 of shoulder abduction from five subjects (except for subject 1 and subject 3 ) is higher than that of

256

257

258

259

260

261

262

263

264

265

266

267

268

269

270

271

272

273

274

275

276

277

278

279

280

281

282

283

284

285

286

287

288

shoulder extension and flexion from Fig. 4. From the above results, we can consider the three classes of MI tasks can be effectively recognized by the proposed method, and shoulder abduction is easy to classify efficiently.

\subsection{Verification of Feature Extraction Capability}

To validate the extraction capability of the proposed feature extraction method, we compared LMD-CSP with some widely used feature extraction methods, including TDP (Tavakolan et al., 2017), CSP (Selim et al., 2018), FBCSP (Ang et al., 2008), and EMD-CSP (Wang et al., 2008). In our work, we used TDP, CSP, FBCSP, EMD-CSP, and LMD-CSP on our data sets for feature extraction, and the same classifier (MOGWO-TWSVM) was employed for recognition. The recognition results are shown in Fig. 6. The average classification accuracy of LMD-CSP $(91.27 \% \pm 5.16 \%)$ is higher than that of TDP $(65.95 \% \pm 13.97 \%)$, CSP $(77.62 \% \pm 4.79 \%)$, FBCSP $(80.47 \% \pm 4.97 \%)$ and EMD-CSP $(84.81 \% \pm 3.92 \%)$. Through analyzing the recognition rate of each subject, we find that LMD-CSP is higher than the compared methods among all subjects. And a one-way ANOVA revealed that there was a statistically significant difference in mean classification accuracy between at least two feature extraction methods $(\mathrm{F}(4,30)=[10.331], p$ value $<0.001)$. Pair-wise t-tests were further applied to calculate $p$-values between LMD-CSP and the other four feature extraction methods. The results are listed in Table 4. Combined the analysis of Fig. 6 and Table 4, show that LMD-CSP has stronger feature extraction capability than the other methods.

Because each MI task activated the corresponding region of the cortex, we further analyzed the contribution of different channels in the classification of the MI tasks. Since there were 3class MI tasks, we obtained three pairs of MI tasks: abduction and extension (A/E), abduction and flexion (A/F), and extension and flexion (E/F). For each pair of MI tasks, the channel weight scores were calculated to highlight the maximally discriminable channels. The weight score of each channel is defined as the ratio of the 2-norm of the corresponding column vector of the CSP filter to the 2-norm of the CSP filter. More details can be seen at Zhou et al. (2015). It should be noted that the weight scores were averaged across subjects and then normalized into the range $(0$, 1). The obtained results are shown in Fig. 7. As can be seen from Fig.7, the prefrontal channels (AF3 and AF4), frontal channels (F3 and F4), and temporal channels (P8 and T8) contribute greatly to the classification for almost each pair of MI tasks. In Tavakolan et al. (2017), the authors stated that the MI tasks of the same limb activated the adjacent cortex. Since the three MI tasks decoded in this paper belong to the same limb, these results are expected.

3.3 Verification of Classification Performance

PeerJ reviewing PDF | (2021:04:60276:3:0:NEW 26 Jul 2021) 
To demonstrate the performance of the proposed classifier, we input the MI feature vectors extracted by LMD-CSP into LDA, ELM, KNN, SVM, LS-SVM, and MOGWO-TWSVM for classification. The obtained results are shown in Fig. 8. It can be seen from Fig. 8 that when compared with the above conventional classifiers, the average classification accuracy by MOGWO-TWSVM is improved, increasing by $18.48 \%, 14.68 \%, 10.24 \%, 7.07 \%$ and $9.52 \%$, respectively. And the difference between the average accuracy of the different classifiers was significant $(\mathrm{F}(5,36)=[9.067], p$-value $<0.001)$. The $p$-values between MOGWO-TWSVM and the other five classifiers were further calculated based on pair-wise t-tests. The results are listed in Table 5. Combined the analysis of Fig. 8 and Table 5, show that MOGWO-TWSVM has good recognition performance and strong robustness.

\subsection{Comparison with Other Recent Methods}

301

302

To demonstrate the validity of the proposed framework, we applied the proposed framework (LMD-CSP and MOGWO-TWSVM) and other recent methods such as the temporal filter parameter optimization with CSP (TFPO-CSP) (Kumar \& Sharma, 2018) and the frequencybased deep learning scheme for recognizing brain wave signals (OPTICAL+) (Kumar, Sharma \& Sharma, 2021) on the same data sets, this data sets include the EEG data of shoulder abduction and extension. The obtained results are shown in Fig. 9. It can be seen that the mean classification accuracy of the proposed framework $(94.76 \% \pm 5.15 \%)$ is higher than that of TFPOCSP $(86.07 \% \pm 10.69 \%)$ and OPTICAL+ $(84.64 \% \pm 15.30 \%)$. And the average recognition rate differed significantly by framework $(\mathrm{F}(2,18)=[3.694], p$-value $=0.045)$. Pair-wise $\mathrm{t}$-tests were further applied for multiple comparisons. The $p$-values between the proposed framework and TFPO-CSP and OPTICAL+ were all less than 0.05 . The above results confirm the validity of the proposed method.

\section{Discussion}

In this paper, three MI tasks of the same joint are successfully recognized by using LMD-CSP and MOGWO-TWSVM with seven healthy subjects. In this section, we will discuss the proposed framework in terms of MI tasks, feature extraction, classification, limitations, and future research lines.

There are few studies on detecting the MI of different movements within the same joint. Therefore, we can only discuss studies that are similar in content to our study. Tavakolan et al. classified three different states (grasp MI, elbow MI, and rest) with an average classification accuracy of $74.2 \%$, using time domain parameters (TDP) including autoregressive model coefficients, root mean square, and waveform length as features and an SVM classifier (Tavakolan et al., 2017). The weakness of that study is that it only identified different joints within the same limb. Mammone et al. reported an average accuracy of $62.47 \%$ for classifying various movements (elbow flexion/extension, forearm pronation/supination, and hand open/close), using time-frequency (TF) maps as features and a deep convolutional neural network (CNN) (Mammone, leractitano, \& Morabito, 2020). However, multi-class MI recognition of the same joint was not involved. In this study, we discriminated 3-class MI tasks of the same joint by using LMD-CSP for feature extraction and MOGWO-TWSVM for classification, the 
329

330

331

332

333

334

335

336

337

338

339

340

341

342

343

344

345

346

347

348

349

350

351

352

353

354

355

356

357

358

359

360

361

362

363

364

365

366

367

368

classification accuracy in seven subjects was no less than $84.44 \%$. Compared with other research, we have investigated the classification of the same joint multi-class MI, and obtained higher recognition accuracy. Due to the success of discriminating single joint MI tasks, more control commands can be provided for BCI systems. And, users can intuitively control external devices, such as a robotic arm, which is of great significance for developing high-performance BCI systems.

The traditional CSP, which is widely applied to extract MI features, requires a lot of electrodes and lacks frequency information. To make up for the lack of frequency information, wavelet packet decomposition (WPD) and CSP were combined to extract effective features (Yang et al., 2016). That study achieved an average classification rate of $88.66 \%$ for left and right hand MI tasks. However, the wavelet basic functions need to be set manually. Kumar et al. employed the genetic algorithm (GA) to select filter parameters, which improved the performance of CSP with a classification error rate of 10.19\% (Kumar \& Sharma, 2018). Li et al. combined orthogonal empirical mode decomposition (OEMD) and a bank of FIR filters to enable the extracted CSP features with frequency domain information (Li et al., 2016). The weakness of this study is that the proposed method has high complexity. In this study, a new adaptive timefrequency analysis algorithm, LMD, which has low computation complexity, and the traditional CSP were combined to extract MI features. LMD-CSP not only makes up for the lack of frequency information, but also improves the adaptability of the algorithm.

The purpose of this paper was to propose a framework for advanced feature extraction and classification of 3-class MI tasks within the same joint. Therefore, TWSVM was applied to classify the extracted MI features, because it has high classification speed and generalization ability. And, the parameters of TWSVM were optimized by MOGWO to improve the classification accuracy. Soman et al. classified left and right hand movements with a kappa value of 0.526, using CSP and TWSVM (Soman \& Jayadeva, 2015). The low kappa value that can be contributed to the hyperparameter selection problem of TWSVM was not considered in that study. In another study, Li et al. employed CSP to extract features from left and right hand MI EEG signals, and the extracted features were input into the chaotic particle swarm optimization twin support vector machine (CSPO TWSVM), the mean accuracy was 75.95\% (Li et al., 2017). Compared with the approaches presented in that study, the proposed classifier (MOGWOTWSVM) achieved a high classification accuracy (91.27\%). The reason may be that MOGWO has high convergence and coverage, which can find the optimal parameters of the TWSVM to achieve the best classification performance.

It should be noted that there are some limitations to this study. On the one hand, we classified the MI tasks by using the same frequency band for different subjects in this study, which limited the classification performance of the proposed framework on some subjects. On the other hand, the sample size for each class in our data set was not large, so detailed statistical analyses were not performed.

In future research, we will verify the effectiveness of the proposed framework by using more experimental EEG data. And, we will try to decode multi-class MI tasks of distal joints, such as

PeerJ reviewing PDF | (2021:04:60276:3:0:NEW 26 Jul 2021) 
369 the wrist joint and finger joint. Additionally, to improve classification performance, we will

370 combine EEG and electromyography (EMG) that are related to movement.

\section{Conclusions}

372 This paper proposes a new scheme for MI recognition of the same joint based on LMD-CSP and MOGWO-TWSVM. The proposed method combines LMD and CSP to extract MI features, where the cloud model is introduced to select effective PF components to enhance the separability of features, thereby successfully extracting feature vectors with high discrimination. Secondly, this paper employs MOGWO to tune the hyperparameters of TWSVM to improve the classification performance and generalization ability of the classifier model, and the average recognition accuracy reaches $91.27 \%$. In future work, we will try to apply the proposed method to complex MI task recognition.

\section{Acknowledgements}

We thank all the subjects who participated in the experiment. We thank Kai Zhao for his guidance on the EEG data acquisition.

\section{Human Ethics}

The following information was supplied relating to ethical approvals: The Scientific Research Ethics and Technology Safety committee of Northeast Electric Power University endorsed the study.

\section{References}

Ang K, Chin Z, Zhang H, Guan C. 2008. Filter bank common spatial pattern (FBCSP) in braincomputer interface. In: 2008 IEEE International Joint Conference on Neural Networks. 23902397.

Aljalal M, Djemal R, Ibrahim S. 2019. Robot Navigation Using a Brain Computer Interface Based on Motor Imagery. Journal of Medical and Biological Engineering 39(4):508-522 DOI 10.1007/s40846-018-0431-9.

\section{Blankertz B, Sannelli C, Haider S, Hammer EM, Kubler A, Muller KR, Curio G, Dickhaus}

T. 2010. Neurophysiological predictor of SMR-based BCI performance. Neuroimage 51(4):13031309 DOI 10.1016/j.neuroimage.2010.03.022.

Bashar SK, Hassan AR, Bhuiyan MIH. 2015. Identification of motor imagery movements from EEG signals using dual tree complex wavelet transform. In: 2015 International Conference on Advances in Computing, Communications and Informatics (ICACCI). 290-296.

Dilip L, Bhesdadiya R, Trivedi I, Jangir P. 2018. Optimal Power Flow Problem Solution Using Multi-objective Grey Wolf Optimizer Algorithm. Lecture Notes in Networks and Systems 19:191201 DOI 10.1007/978-981-10-5523-2_18.

Delgado JMC, Achanccaray D, Villota ER, Chevallier S. 2020. Riemann-Based Algorithms Assessment for Single- and Multiple-Trial P300 Classification in Non-Optimal Environments. IEEE Transactions on Neural Systems and Rehabilitation Engineering 28(12):2754-2761 DOI 10.1109/TNSRE.2020.3043418.

Edelman BJ, Baxter B, He B. 2016. EEG source imaging enhances the decoding of complex right hand motor imagery tasks. IEEE Transactions on Biomedical Engineering 63(1):4-14 DOI 10.1109/TBME.2015.2467312.

Hinterberger T, Schmidt S, Neumann N, Mellinger J, Blankertz B, Curio G, Birbaumer N. 2004. Brain-computer communication and slow cortical potentials. IEEE Transactions on 
412

413

414

415

416

417

418

419

420

421

422

423

424

425

426

427

428

429

430

431

432

433

434

435

436

437

438

439

440

441

442

443

444

445

446

447

448

449

450

451

452

453

454

455

456

457

458

Biomedical Engineering 51(6):1011-1018 DOI 10.1109/TBME.2004.827067.

Jayadeva, Khemchandani R, Chandra S. 2007. Twin support vector machines for pattern classification. IEEE Transactions on Pattern Analysis and Machine Intelligence 29(5):905-910 DOI 10.1109/TPAMI.2007.1068.

Kumar S, Sharma A. 2018. A new parameter tuning approach for enhanced motor imagery EEG signal classification. Medical \& Biological engineering \& Computing 56(10):1861-1874 DOI 10.1007/s11517-018-1821-4.

Kumar S, Sharma R, Sharma A. 2021. OPTICAL+: a frequency-based deep learning scheme for recognizing brain wave signals. Peerj computer science 7:e375 DOI 10.7717/peerj-cs.375 Liu C, Yan S, Zhao H, Wang H. 2012. Study on multi-class motor imagery EEG classification based on KNN. Chinese Journal of Scientific Instrument 33(8):1714-1720 DOI 10.19650/j.cnki.cjsi.2012.08.005.

Li M, Guo S, Yang J, Sun Y. 2016. A novel EEG feature extraction method based on OEMD and CSP algorithm. Journal of Intelligent \& Fuzzy Systems 30(5):2971-2983 DOI 10.3233/IFS151896.

Li D, Zhang H, Khan MS, Mi F. 2017. Recognition of motor imagery tasks for BCI using CSP and chaotic PSO twin SVM. The Journal of China Universities of Posts and Telecommunications 24(3):83-90 DOI 10.1016/S1005-8885(17)60215-2.

Li M, Dong Y, Yang J, Duan L. 2020. Subject-based dipole selection for decoding motor imagery tasks. Neurocomputing 402:195-208. DOI 10.1016/j.neucom.2020.03.055.

Mirjalili S, Saremi S, Mirjalili SM, Coelho LD. 2016. Multi-objective grey wolf optimizer: A novel algorithm for multi-criterion optimization. Expert Systems with Applications 47:106-119 DOI 10.1016/j.eswa.2015.10.039.

Miao M, Wang A, Liu F. 2017. A spatial-frequency-temporal optimized feature sparse representation-based classification method for motor imagery EEG pattern recognition. Medical \& Biological Engineering \& Computing 55(9):1589-1603

Malan NS, Sharma S. 2019. Feature selection using regularized neighbourhood component analysis to enhance the classification performance of motor imagery signals. Computers in Biology and Medicine 107:118-126 DOI 10.1016/j.compbiomed.2019.02.009.

Mammone N, Ieracitano C, Morabito FC. 2020. A deep CNN approach to decode motor preparation of upper limbs from time-frequency maps of EEG signals at source level. Neural networks 124:357-372 DOI 10.1016/j.neunet.2020.01.027.

Park Y, Chung W. 2020. A novel EEG correlation coefficient feature extraction approach based on demixing EEG channel pairs for cognitive task classification. IEEE Access 8:87422-87433 DOI 10.1109/ACCESS.2020.2993318.

Rodriguez-Bermudez G, Bueno-Crespo A, Martinez-Albaladejo FJ. 2017. Classifying BCI signals from novice users with extreme learning machine. Open Physics, 15(1):494-500 DOI 10.1515/phys-2017-0056.

Smith JS. 2005. The local mean decomposition and its application to EEG perception data. Journal of the Royal Society Interface 2(5):443-454 DOI 10.1098/rsif.2005.0058.

Soman S, Jayadeva. 2015. High performance EEG signal classification using classifiability and the Twin SVM. Applied Soft Computing 30:305-318 DOI 10.1016/j.asoc.2015.01.018.

Selim S, Tantawi MM, Shedeed HA, Badr A. 2018. A CSP/AM-BA-SVM approach for motor imagery BCI system. IEEE Access 6:49192-49208 DOI 10.1109/ACCESS.2018.2868178.

Tavakolan M, Frehlick Z, Yong X, Menon C. 2017. Classifying three imaginary states of the same upper extremity using time-domain features. Plos One 12(3):e0174161

DOI 10.1371/journal.pone.0174161.

PeerJ reviewing PDF | (2021:04:60276:3:0:NEW 26 Jul 2021) 
459

460

461

462

463

464

465

466

467

468

469

470

471

472

473

474

475

476

477

478

479

480

481

482

483

484

485

486

487

488

489

490

491

492

493

Taran S, Bajaj V, Sharma D, Siuly S, Sengur A. 2018. Features based on analytic IMF for classifying motor imagery EEG signals in $\mathrm{BCI}$ applications. Measurement 116:68-76 DOI 10.1016/j.measurement.2017.10.067.

Taran S, Bajaj V. 2019. Motor imagery tasks-based EEG signals classification using tunable-Q wavelet transform. Neural computing \& applications 31(11):6925-6932

DOI 10.1007/s00521-018-3531-0.

Vuckovic A, Sepulveda F. 2012. A two-stage four-class BCI based on imaginary movements of the left and the right wrist. Medical Engineering and Physics 34(7):964-971

DOI 10.1016/j.medengphy.2011.11.001.

Wang Z, Maier A, Logothetis NK, Liang H. 2008. Single-Trial Classification of Bistable Perception by Integrating Empirical Mode Decomposition, Clustering, and Support Vector Machine. EURASIP Journal on Advances Signal Processing 2008:592742 DOI 10.1155/2008/592742.

Wang S, Chi H, Yuan Z, Geng J. 2019. Emotion Recognition Using Cloud Model. Chinese Journal of Electronics 28(3):470-474 DOI 10.1049/cje.2018.09.020.

Wang H, Xu T, Tang C, Yue H, Chen C, Xu L, Pei Z, Dong J, Bezerianos A, Li J. 2020. Diverse Feature Blend Based on Filter-Bank Common Spatial Pattern and Brain Functional Connectivity for Multiple Motor Imagery Detection. IEEE Access 8:155590-155601 DOI 10.1109/access.2020.3018962.

Yang B, Li H, Wang Q, Zhang, Y. 2016. Subject-based feature extraction by using fisher WPDCSP in brain-computer interfaces. Computer Methods Programs Biomed 129:21-28 DOI 10.1016/j.cmpb.2016.02.020.

You Y, Chen W, Zhang W. 2020. Motor imagery EEG classification based on flexible analytic wavelet transform. Biomedical Signal Processing and Control 62:102069

DOI 10.1016/j.bspc.2020.102069.

Zhou B, Wu X, Lu Z, Zhang L, Guo X, Zhang C. 2015. Channel selection for multi-class motor imagery based on common spatial pattern. Journal of biomedical engineering 32(3):520525 DOI 10.7507/1001-5515.20150095.

Zhang T, Chen W. 2017. LMD based features for the automatic seizure detection of EEG signals using SVM. IEEE Transactions on Neural Systems and Rehabilitation Engineering. 25(8):11001108 DOI 10.1109/TNSRE.2016.2611601.

Zhou Y, He S, Huang Q, Li Y. 2020. A hybrid asynchronous brain-computer interface combining SSVEP and EOG signals. IEEE Transaction on Biomedical Engineering 67(10):28812892 DOI 10.1109/TBME.2020.2972747.

Peer) reviewing PDF | (2021:04:60276:3:0:NEW 26 Jul 2021) 


\section{Table $\mathbf{1}$ (on next page)}

Parameter setting used in multi-objective grey wolf optimization (MOGWO). 
1 Table 1:

2 Parameter setting used in multi-objective grey wolf optimization (MOGWO).

\begin{tabular}{ccc}
\hline Parameter & Name & Value \\
\hline$n$ & Number of wolves & 12 \\
$M_{\mathrm{I}}$ & Max iterations & 100 \\
$A$ & Archive size & 10 \\
$\alpha$ & Grid inflation parameter & 0.1 \\
$\beta$ & Leader selection parameter & 4 \\
$\delta$ & Number of grids per dimension & 10 \\
\hline
\end{tabular}

3 


\section{Table 2 (on next page)}

Pareto optimal set of subject 4 and the corresponding parameters of twin support vector machine (TWSVM). 
1 Table 2:

2 Pareto optimal set of subject 4 and the corresponding parameters of twin support vector machine

3 (TWSVM).

\begin{tabular}{ccccccc}
\hline $\boldsymbol{c}_{\mathbf{1}}$ & $\boldsymbol{c}_{\mathbf{2}}$ & $\boldsymbol{\lambda}$ & $\boldsymbol{C R}_{\mathbf{1}}$ & $\boldsymbol{C R}_{\mathbf{2}}$ & $\boldsymbol{C R}_{\mathbf{3}}$ & Accuracy \\
\hline 2.2342 & 4.3876 & 3.8087 & 1.00 & 0.80 & 0.80 & 0.8667 \\
2.6221 & 1.8131 & 5.2273 & 0.90 & 0.90 & 0.85 & 0.8833 \\
2.6688 & 0.7101 & 4.7547 & 0.85 & 0.65 & 0.90 & 0.8000 \\
2.1949 & 1.1183 & 0.1383 & 0.35 & 0.15 & 1.00 & 0.5000 \\
2.3311 & 1.3203 & 3.5416 & 1.00 & 0.75 & 0.85 & 0.8667 \\
$\mathbf{2 . 3 2 2 9}$ & $\mathbf{1 . 7 5 3 9}$ & $\mathbf{4 . 3 8 9 0}$ & $\mathbf{0 . 9 5}$ & $\mathbf{0 . 8 5}$ & $\mathbf{0 . 8 5}$ & $\mathbf{0 . 8 8 3 3}$ \\
2.4102 & 0.8047 & 5.4034 & 0.65 & 0.70 & 0.90 & 0.7500 \\
2.6035 & 1.8690 & 4.6250 & 0.95 & 0.90 & 0.80 & 0.8833 \\
2.1782 & 0.7592 & 5.2358 & 0.60 & 0.70 & 0.95 & 0.7500 \\
\hline
\end{tabular}

4 
Table 3(on next page)

Averaged precision and recall of three class motor imagery (MI) tasks under the proposed method. 
1 Table 3:

2 Averaged precision and recall of three class motor imagery (MI) tasks under the proposed 3 method.

\begin{tabular}{ccc}
\hline Subject & Precision (\%) & Recall (\%) \\
\hline subject 1 & 96.75 & 96.67 \\
subject 2 & 91.55 & 91.11 \\
subject 3 & 85.15 & 84.44 \\
subject 4 & 88.65 & 88.33 \\
subject 5 & 93.45 & 93.33 \\
subject 6 & 87.05 & 86.67 \\
subject 7 & 98.41 & 98.33 \\
average & 91.57 & 91.27 \\
\hline
\end{tabular}

4 


\section{Table 4 (on next page)}

Paired t-test ( $p$-value) between the proposed feature extraction method (LMD-CSP) and the other four feature extraction methods. 
1 Table 4:

2 Paired t-test ( $p$-value) between the proposed feature extraction method (LMD-CSP) and the other

3 four feature extraction methods.

\begin{tabular}{ccccc}
\hline Method & TDP & CSP & FBCSP & EMD-CSP \\
\hline$p$-value & 0.0022 & 0.0001 & 0.0013 & 0.0141 \\
\hline
\end{tabular}

4 


\section{Table 5 (on next page)}

Paired t-test ( $p$-value) between multi-objective grey wolf optimization twin support vector machine (MOGWO-TWSVM) and the other five classifiers. 
1 Table 5:

2 Paired t-test ( $p$-value) between multi-objective grey wolf optimization twin support vector

3 machine (MOGWO-TWSVM) and the other five classifiers.

\begin{tabular}{cccccc}
\hline Method & LDA & ELM & KNN & SVM & LS-SVM \\
\hline$p$-value & 0.0002 & 0.0033 & 0.0009 & 0.0005 & 0.0186 \\
\hline
\end{tabular}

4 
Figure 1

Emotive Epoc+ and the placement position of Emotive electrodes.

(A) Emotive Epoc+ EEG signal acquisition instrument. (B) Location of all electrodes and marked electrodes are used in this study.

A

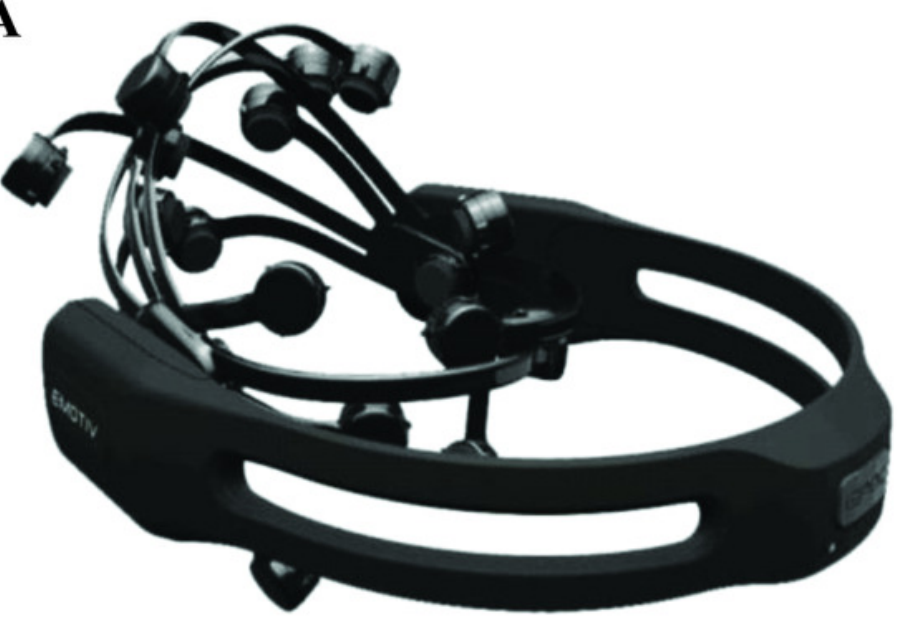

B

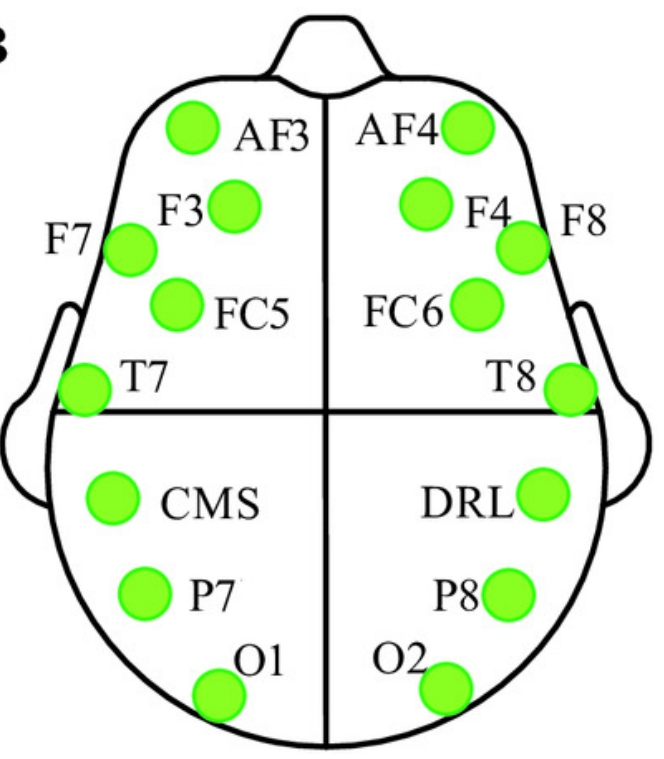


Figure 2

Experimental paradigm for the motor imagery (MI) tasks.

(A) The experiment included three MI tasks (shoulder abduction, flexion, and extension). (B) The experimental process of one trail.

A

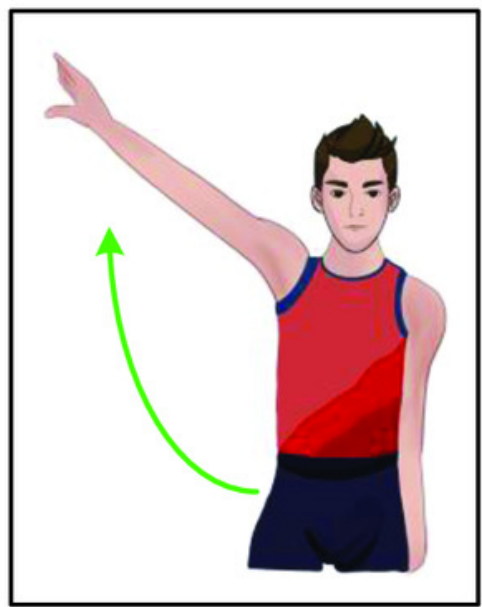

Abduction

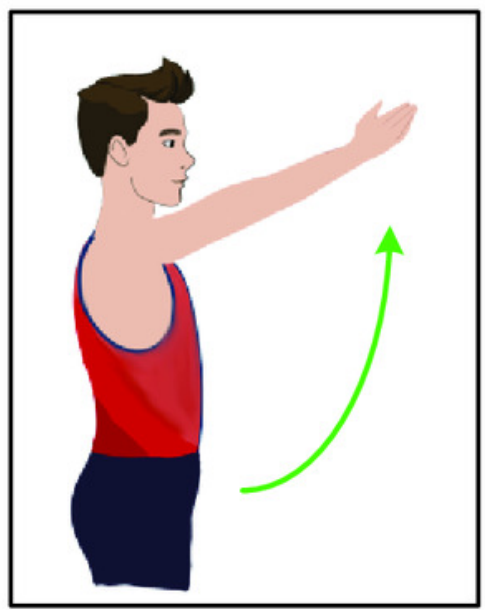

Flexion

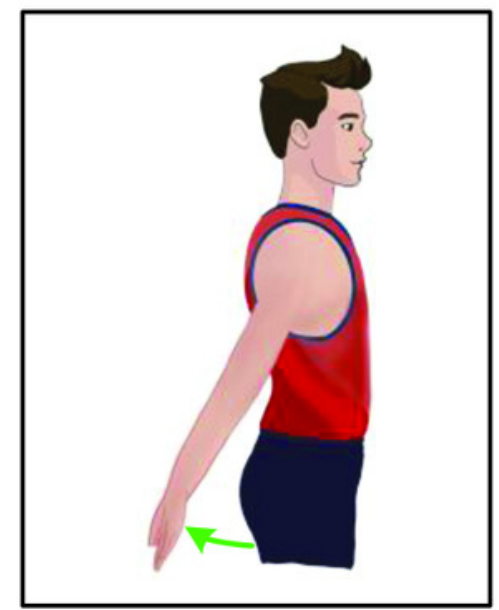

Extension

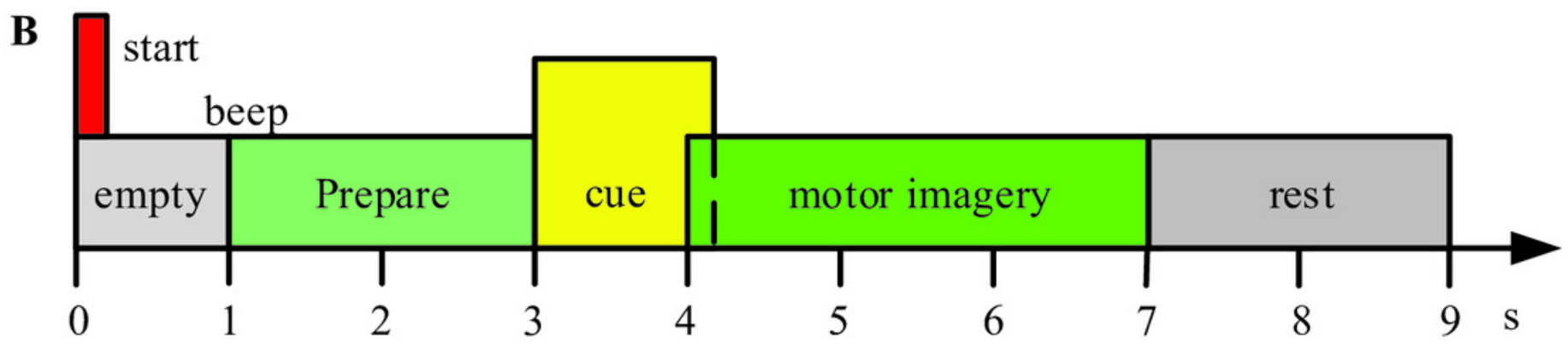


Figure 3

Distributions of the best two features obtained by the proposed feature extraction method (LMD-CSP).

(A) Distribution of the best two features of abduction and extension. (B) Distribution of the best two features of abduction and flexion. (C) Distribution of the best two features of extension and flexion.
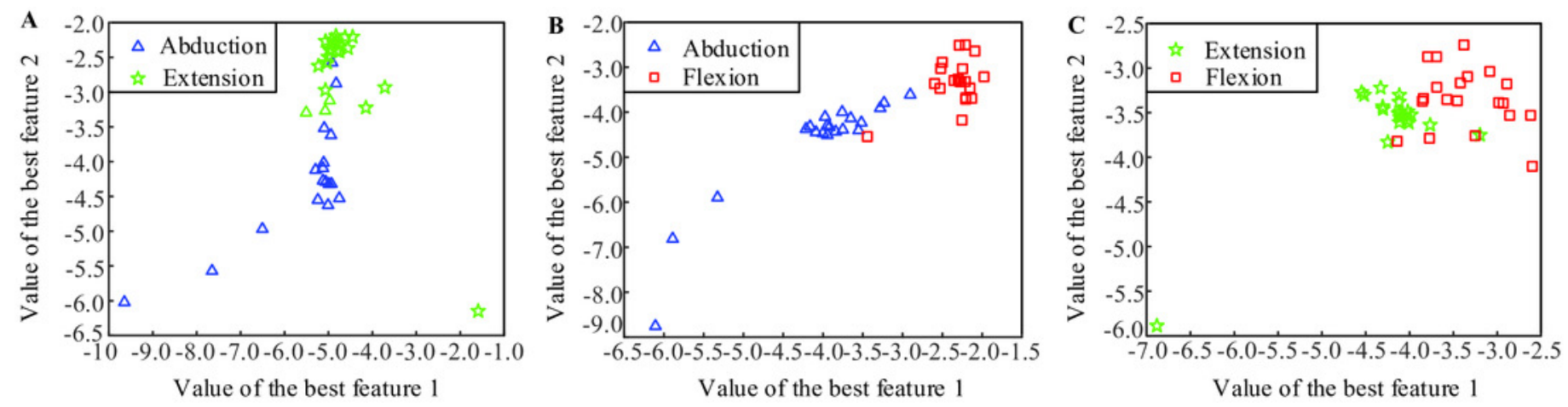
Figure 4

Average classification accuracies for the three categories.

Three motor imagery (MI) tasks classification accuracy of all subjects obtained using the proposed method (LMD-CSP and MOGWO-TWSVM).

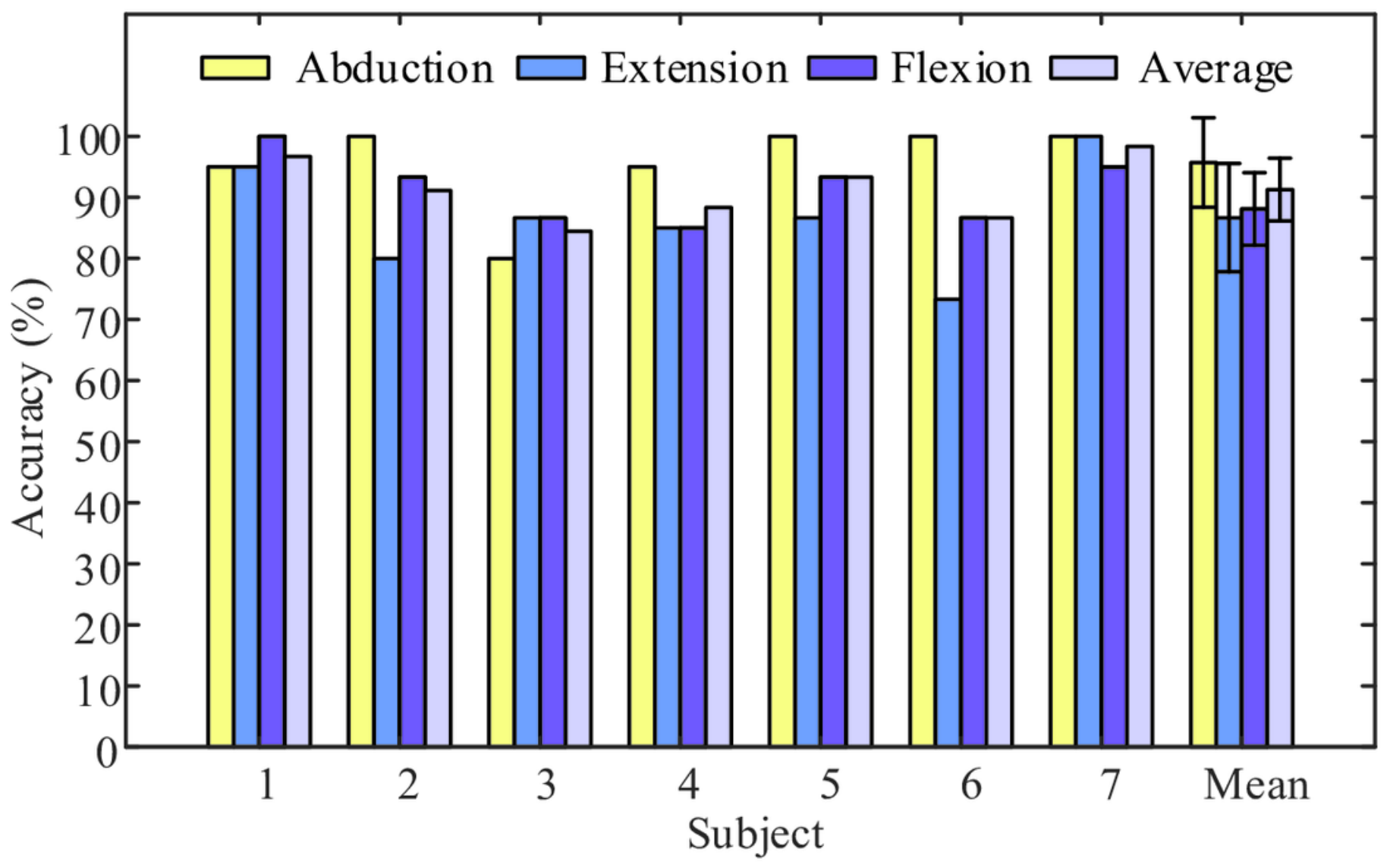


Figure 5

The mean confusion matrix of all subjects (Abdu represents abduction, Ext represents extension, Flex represents flexion). 
Abdu Ext Flex

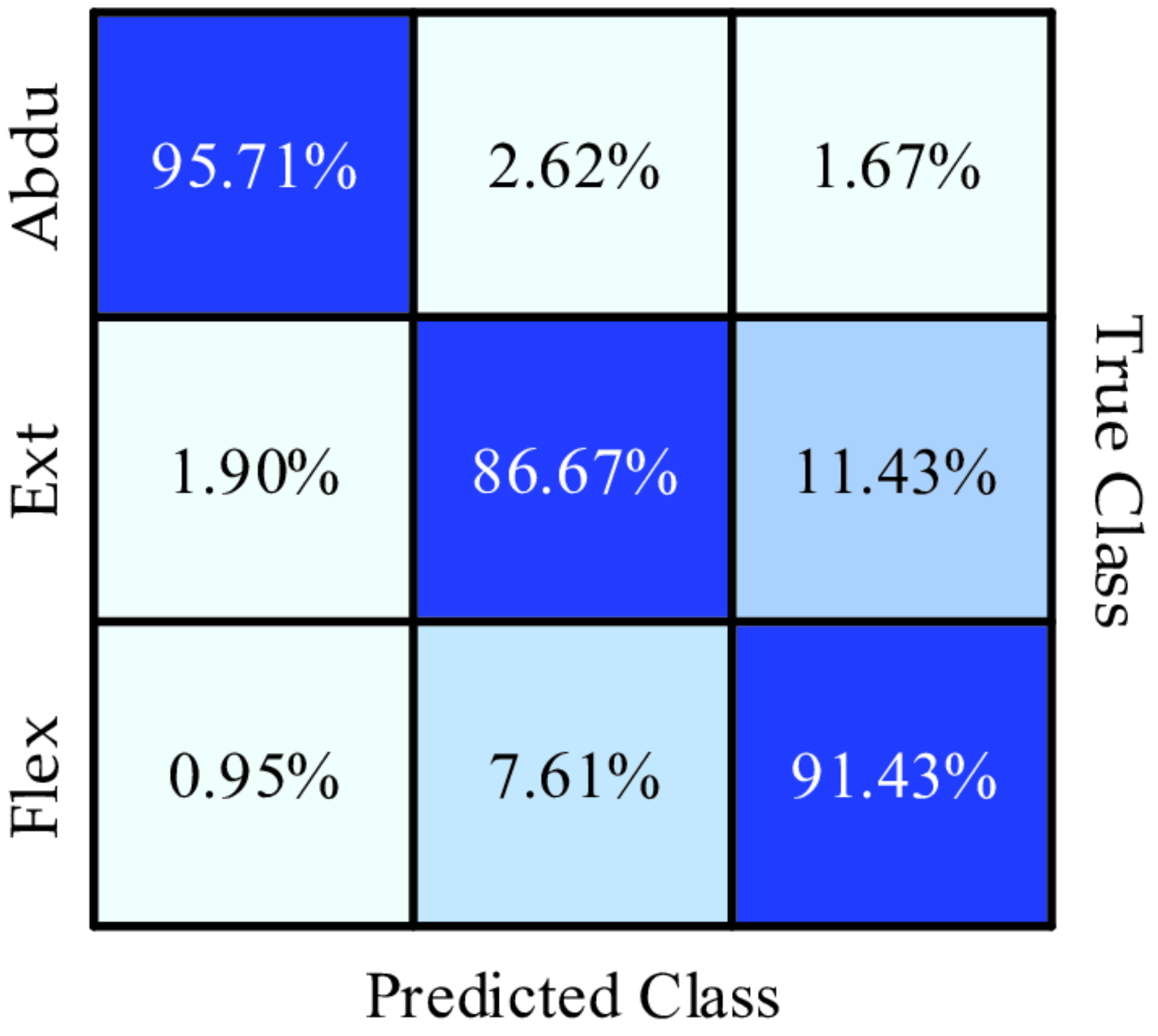

$0 \%$

$100 \%$ 


\section{Figure 6}

Comparison of classification accuracy obtained by the different feature extraction methods.

Five different feature extraction methods, including time domain parameters (TDP), common spatial pattern (CSP), filter-bank common spatial pattern (FBCSP), common spatial pattern based on empirical mode decomposition (EMD-CSP), and the proposed feature extraction method (LMD-CSP), were employed to extract motor imagery (MI) features on our data sets, respectively. Then, the same multi-objective grey wolf optimization twin support vector machine (MOGWO-TWSVM) classified those MI features to obtain classification accuracy of all subjects.

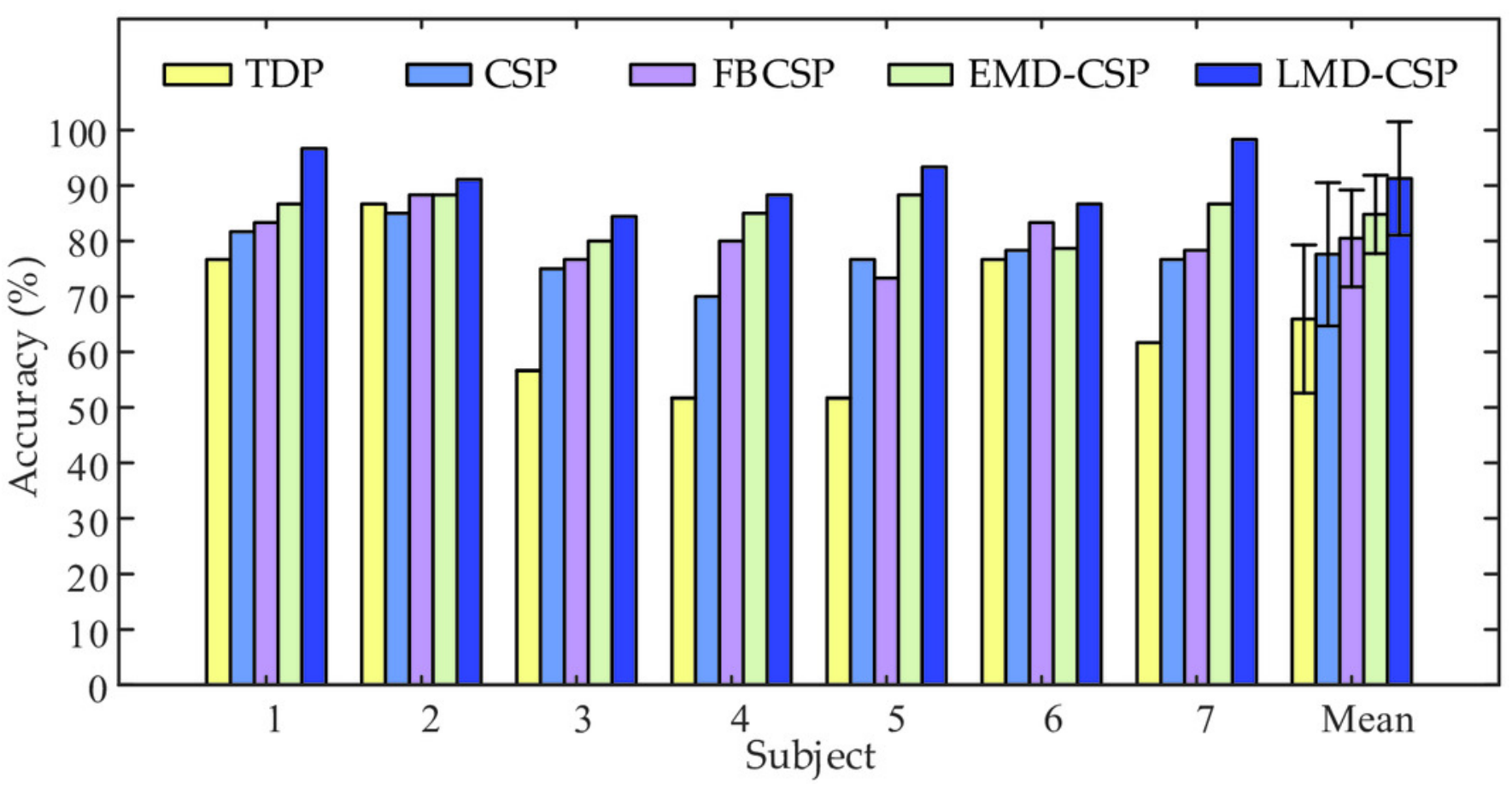




\section{Figure 7}

Averaged channel weight scores for each pair of motor imagery tasks ( $A / E$ represents abduction and extension, A/F represents abduction and flexion, and E/F represents extension and flexion).

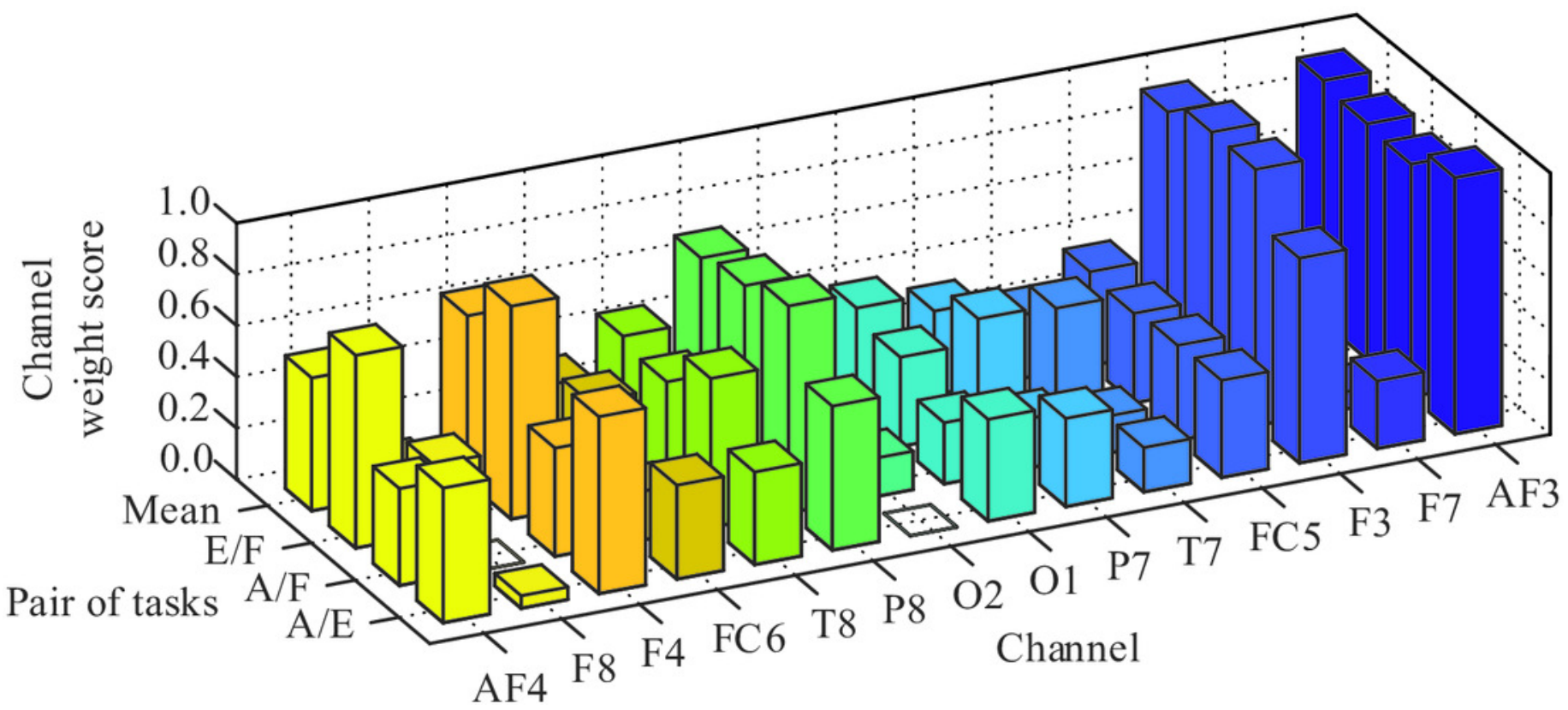




\section{Figure 8}

Comparison of classification accuracy obtained by the different classifiers.

The proposed feature extraction method (LMD-CSP) was employed to extract (motor imagery) Ml features on our data sets. Then the same features were classified by six different classifier, including linear discriminant analysis (LDA), extreme learning machine (ELM), knearest neighbors (KNN), least squares support vector machine (LS-SVM), and the proposed classifier (MOGWO-TWSVM), to obtain classification accuracy of all subjects.

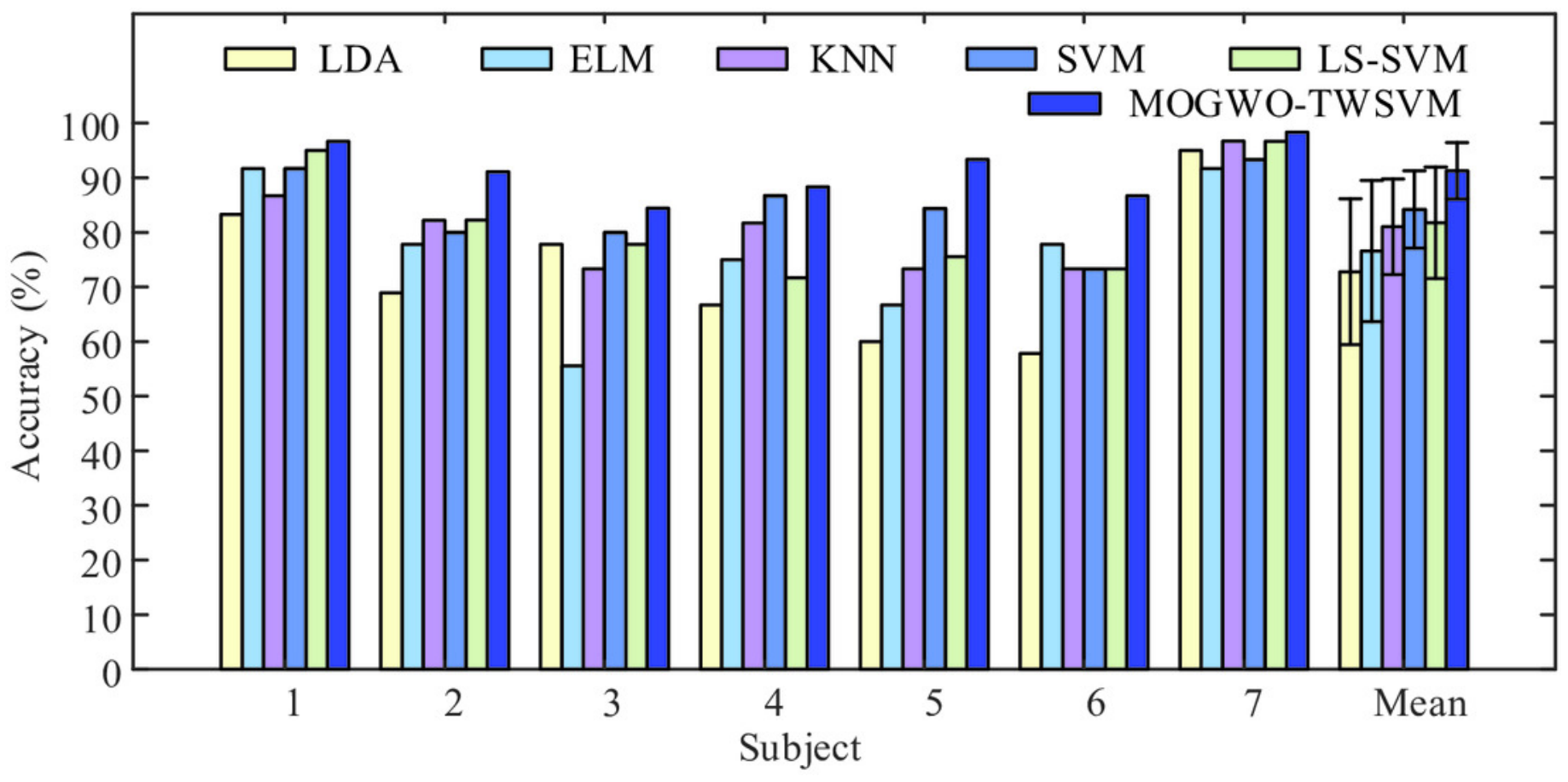




\section{Figure 9}

Classification accuracy comparison by the proposed method with other recent methods.

The temporal filter parameter optimization with CSP (TFPO-CSP), the frequency-based deep learning scheme for recognizing brain wave signals (OPTICAL+), and the proposed method were employed to discriminate two-class EEG data (shoulder abduction and extension) in our data sets to obtain the classification accuracy of all subjects.

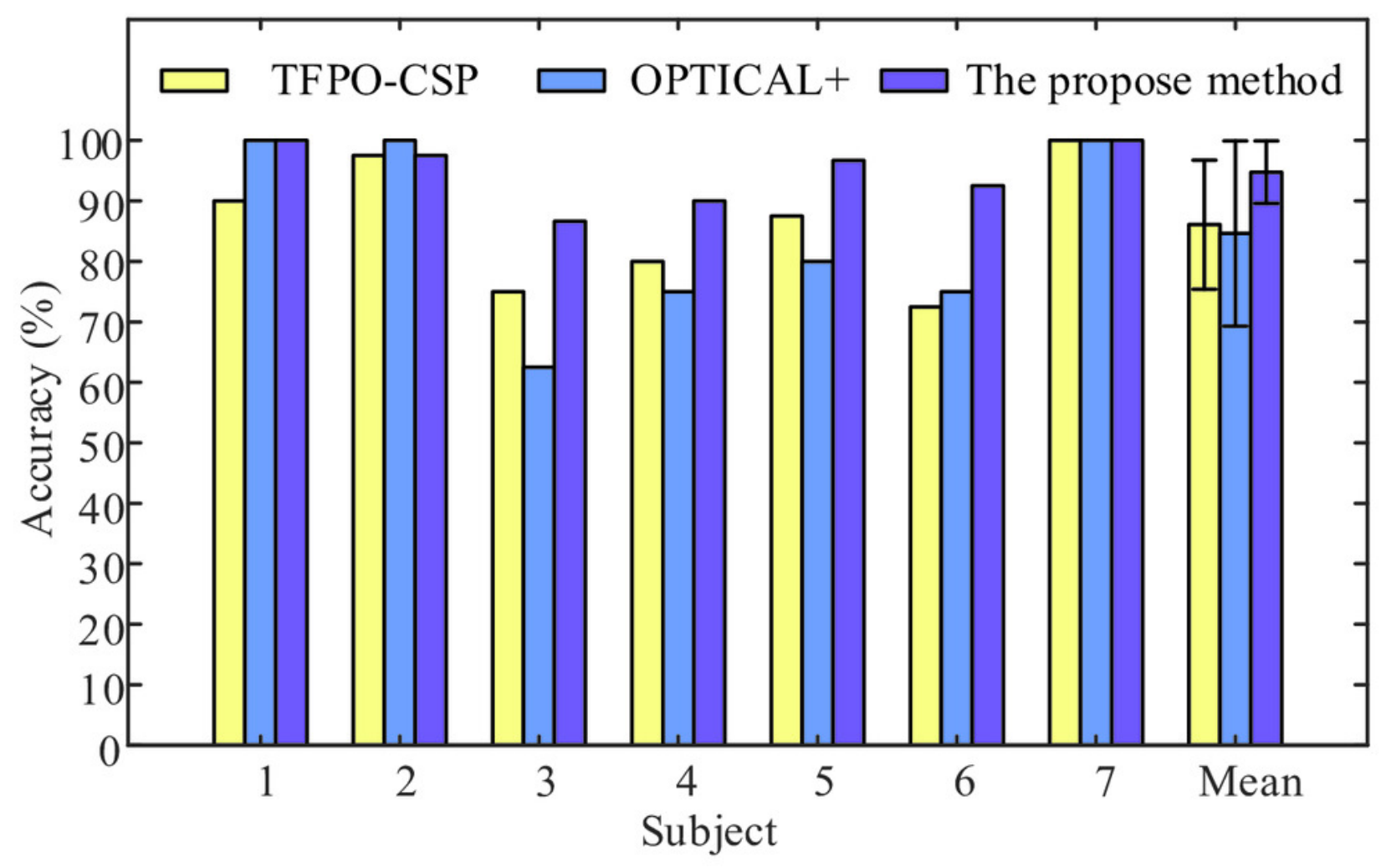

